\title{
3D-2D image registration by nonlinear regression
}

\section{Author(s)}

Gouveia, A.R.

CICS-UBI - Health Sci. Res. Centre, Univ. of Beira Interior, Covilhã, Portugal

Metz, C. ; Freire, L. ; Klein, S.

\section{Abstract}

We propose a 3D-2D image registration method that relates image features of 2D projection images to the transformation parameters of the 3D image by nonlinear regression. The method is compared with a conventional registration method based on iterative optimization. For evaluation, simulated X-ray images (DRRs) were generated from coronary artery tree models derived from 3D CTA scans. Registration of nine vessel trees was performed, and the alignment quality was measured by the mean target registration error (mTRE). The regression approach was shown to be slightly less accurate, but much more robust than the method based on an iterative optimization approach.

\section{Published in:}

Biomedical Imaging (ISBI), 2012 9th IEEE International Symposium on

Date of Conference: 2-5 May 2012

Page(s):

$1343-1346$

ISSN :

$1945-7928$

Print ISBN:

978-1-4577-1857-1

INSPEC Accession Number:

12864598

Conference Location :

Barcelona

Digital Object Identifier :

10.1109/ISBI.2012.6235814 


\section{AUTHOR KEYWORDS}

- $2 \mathrm{D} / 3 \mathrm{D}$ image registration

- image guided interventions

- regression

\section{IEEE TERMS}

- Feature extraction

- Image registration

- Neural networks

- Optimization

- Robustness

- Training

- X-ray imaging 